Article

\title{
Adaptive Square-Root Unscented Kalman Filter-Based State-of-Charge Estimation for Lithium-Ion Batteries with Model Parameter Online Identification
}

\author{
Quan Ouyang, Rui Ma, Zhaoxiang $\mathrm{Wu}$, Guotuan $\mathrm{Xu}$ and Zhisheng Wang * \\ College of Automation Engineering, Nanjing University of Aeronautics and Astronautics, \\ Nanjing 210016, China; ouyangquan@nuaa.edu.cn (Q.O.); maruinuaa@nuaa.edu.cn (R.M.); \\ wuzhaoxiang@nuaa.edu.cn (Z.W.); xuguotuan@126.com (G.X.) \\ * Correspondence: wangzhisheng@nuaa.edu.cn; Tel.: +86-025-5283-2301
}

Received: 13 August 2020; Accepted: 17 September 2020; Published: 22 September 2020

check for updates

\begin{abstract}
The state-of-charge (SOC) is a fundamental indicator representing the remaining capacity of lithium-ion batteries, which plays an important role in the battery's optimized operation. In this paper, the model-based SOC estimation strategy is studied for batteries. However, the battery's model parameters need to be extracted through cumbersome prior experiments. To remedy such deficiency, a recursive least squares (RLS) algorithm is utilized for model parameter online identification, and an adaptive square-root unscented Kalman filter (SRUKF) is designed to estimate the battery's SOC. As demonstrated in extensive experimental results, the designed adaptive SRUKF combined with RLS-based model identification is a promising SOC estimation approach. Compared with other commonly used Kalman filter-based methods, the proposed algorithm has higher precision in the SOC estimation.
\end{abstract}

Keywords: lithium-ion batteries; state-of-charge estimation; adaptive square-root unscented Kalman filter; recursive least squares

\section{Introduction}

Rechargeable lithium-ion batteries have been widely used in numerous applications due to their superior power performance, long life cycle, and so forth [1,2]. The state-of-charge (SOC) is an important and fundamental parameter that indicates the remaining available capacity of the battery [3]. Inaccurate SOC estimation can easily lead to the battery's overdischarge or overcharge even resulting in an explosion. It hence calls for promising strategies to accurately estimate the SOC of the battery's.

Two commonly utilized non-model-based SOC estimation approaches are the ampere-hour counting algorithm and open circuit voltage (OCV) method, respectively. Ampere-hour counting algorithm uses the time integration of the current as the indicator of the change of the SOC of the battery. Although this method is simple to implement, measurement errors accumulate since the SOC calculation process is open-loop based. Generally, it needs frequent calibrations to eliminate the battery's SOC estimation error. Derived from the identified relationship between the battery's OCV and SOC [4], the SOC can be obtained through measuring the OCV of the battery. However, the OCV can only be measured by cutting the battery off from the external circuit, which could inconvenience the battery user.

In order to get more accurate estimated SOC, model-based SOC estimation strategies attract great research attention. Currently, the commonly used battery models can be classified into the following three categories: electrochemical mechanism models [5,6], equivalent circuit models [7], 
and neural network models [8]. Among them, the equivalent circuit models, utilizing the circuits to simulate the dynamic characteristics of the battery, have reasonable computational complexity and great flexibility in battery materials and sizes, which are the most suitable to be utilized for the SOC estimation. Extended Kalman filter (EKF) algorithms are the most widely studied model-based SOC estimation approaches [9,10]. Adaptive EKFs are proposed in [11] and [12] to estimate the SOC with noise covariances online update. However, the nonlinear battery model needs to be linearized in these EKF algorithms, which will reduce the SOC estimation accuracy of the battery. The unscented Kalman filter (UKF) [13] and adaptive UKF [14] are proposed as the improvements of the EKFs for the SOC estimation. The first-order linearization is not required in the UKFs. Instead, they utilize a nonlinear transformation, called unscented transform (UT), for the propagation of the battery states' mean and covariance. The square-root UKF (SRUKF) can be treated as an enhanced version of the UKF with the attached advantage that the positive semi-definiteness of the state covariance can be guaranteed [15]. In addition, it needs less computational complexity for state estimation. In [16], based on a radial basis function neural network battery model, an SRUKF is utilized for the SOC estimation. A square-root spherical UKF is designed for SOC estimation for the battery in Nanosatellite [17]. An adaptive SRUKF method is designed in [18] to estimate the battery's SOC. A proportional-integral observer is proposed for the SOC estimation of the battery in electric drive vehicles [19]. A neural network-based SOC observer is proposed in [20] with the convergence proved utilizing Lyapunov stability analysis. A disturbance observer-based strategy is designed in [21] to estimate the SOC, which can reduce the computational burden without reducing the estimation accuracy.

The above SOC estimation strategies [9-14,16-20] all run on a battery model with parameters known. However, the model parameters must be extracted by cumbersome prior experiments. It will be more convenient if the model parameters are not necessary to be identified in advance for the designed SOC estimation algorithm. Therefore, an interesting and important question can be investigated: how to design a battery's SOC estimation strategy with the model parameters treated as unknown values. In [22], a recursive least squares (RLS) algorithm is utilized to determine the model parameters of the battery, and a linear observer is adopted to estimate the battery's SOC. However, the model noise information is not considered in this method, and the SOC estimation accuracy is reduced. The battery's SOC and model parameters are simultaneously estimated by utilizing an iterated EKF in [23]. A UKF algorithm is developed to estimate the SOC with another adaptive UKF utilized for the online identification of the model parameters of the battery in [24]. Its experimental results indicate that this UKF algorithm has higher SOC estimation accuracy compared with the EKF.

In this paper, an equivalent circuit model is utilized to describe the dynamics of the battery, where the model parameters are considered to be unknown. An RLS algorithm is utilized for model parameter online identification. Next, an adaptive SRUKF based on this online identified battery model is developed to estimate the battery's SOC, where a noise statistic estimator is utilized for the noise information online update. The main novelties of this work are summarized in the following two aspects.

(1) The model parameters are treated as unknown variables, and an RLS algorithm is utilized to estimate them online. This can avoid unnecessary experiments prior to SOC estimation for parameter extraction.

(2) Extensive experiments demonstrate the effectiveness of the proposed charging strategy showing that the proposed adaptive SRUKF can provide higher SOC estimation accuracy compared with other commonly used Kalman filter-based methods.

The rest of this manuscript is arranged as follows. In Section 2, the battery's equivalent circuit model is presented. In Section 3, an RLS algorithm is utilized for the online model parameter identification, and an adaptive SRUKF is developed to estimate the SOC of the battery. Related experimental results are included in Section 4, and concluding remarks are provided in Section 5. 


\section{Battery Model Development}

\subsection{Battery Equivalent Circuit Model}

A commonly used equivalent circuit model $[25,26]$ is utilized to simulate the lithium-ion battery's dynamics, which can strike an excellent balance between accuracy and simplicity. As illustrated in Figure 1, the capacitor $C_{b}$ denotes the battery's full charged capacity, and the resistor $R_{0}$ represents the battery's energy losses during the charging and discharging process. The RC network $\left(R_{t}, C_{t}\right)$ characterizes the battery's transient voltage-current response. The voltage across $C_{b}$ represents the battery's SOC quantitatively, with $0-1 \mathrm{~V}$ corresponding to $0-100 \%$ of the SOC [20]. The mapping from the SOC to the OCV of the battery is denoted as

$$
V_{O C}=g(S O C)
$$

where $V_{O C}$ and SOC are the battery's OCV and SOC, respectively; $g(\cdot)$ is a nonlinear function. Based on the Kirchhoff's laws of current and voltage, the dynamics of the battery is obtained as

$$
\begin{aligned}
S \dot{O} C & =-\frac{\eta_{0}}{C_{b}} I_{B} \\
\dot{V}_{t} & =-\frac{1}{R_{t} C_{t}} V_{t}+\frac{1}{C_{t}} I_{B} \\
V_{B} & =V_{O C}-R_{0} I_{B}-V_{t},
\end{aligned}
$$

where $V_{t}, V_{B}$, and $I_{B}$ are, respectively, the voltage across the capacitor $C_{t}$, the terminal voltage, and the current of the battery; $\eta_{0}$ is the Coulomb coefficient.
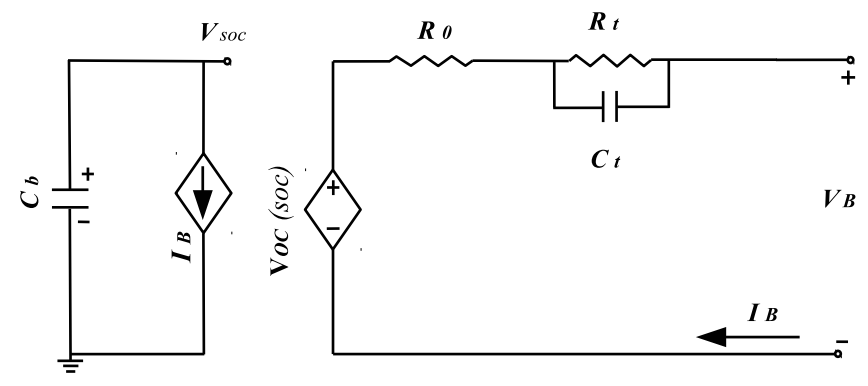

Figure 1. Equivalent circuit model of the battery.

\subsection{Model Discretization}

With the current $I_{B}$ assumed to be constant during each sampling period, and the battery model (2) can be discretized as follows:

$$
\begin{aligned}
\operatorname{SOC}(k) & =\operatorname{SOC}(k-1)-\frac{T}{C_{b}} I_{B}(k-1) \\
V_{t}(k) & =e^{-\frac{T}{R_{t} C_{t}}} V_{t}(k-1)+\left(1-e^{-\frac{T}{R_{t} C_{t}}}\right) R_{t} I_{B}(k-1) \\
V_{B}(k) & =g(\operatorname{SOC}(k))-R_{0} I_{B}(k)-V_{t}(k)
\end{aligned}
$$

with $T$ the sampling period. To simplify the notations, with the process and measurement noise considered, the battery model (3) can be expressed in the following form

$$
\begin{aligned}
& x(k)=A(\theta) x(k-1)+B(\theta) u(k-1)+w(k) \\
& y(k)=h(x(k), \theta, u(k))+v(k)
\end{aligned}
$$


with

$$
\begin{gathered}
A(\theta)=\left[\begin{array}{cc}
1 & 0 \\
0 & \theta_{2}
\end{array}\right], B(\theta)=\left[\begin{array}{c}
-\frac{T}{C_{b}} \\
\theta_{1}-\theta_{1} \theta_{2}
\end{array}\right] \\
h(x(k), \theta, u(k))=g\left(x_{1}(k)\right)-x_{2}(k)-\theta_{3} u(k)
\end{gathered}
$$

where the system output is $y(k) \triangleq V_{B} \in \mathbb{R} ; u(k) \triangleq I_{B} \in \mathbb{R}$ is the system input; the states $x(k) \in \mathbb{R}^{2}$ and parameters $\theta \in \mathbb{R}^{3}$ are defined as $x(k)=\left[x_{1}(k), x_{2}(k)\right]^{T} \triangleq\left[S O C(k), V_{t}(k)\right]^{T}$ and $\theta=\left[\theta_{1}, \theta_{2}, \theta_{3}\right]^{T} \triangleq\left[R_{t}\right.$, $\left.e^{-\frac{T}{R_{t} C_{t}}}, R_{0}\right]^{T}$, respectively; $w(k)$ and $v(k)$ are the process noise and the measurement noise of the battery model, which are usually assumed as uncorrelated zero-mean Gaussian white noises that satisfy

$$
\begin{aligned}
& w(k) \sim N\left(0, Q^{2}\right) \\
& v(k) \sim N\left(0, R^{2}\right),
\end{aligned}
$$

with $Q^{2} \in \mathbb{R}^{2 \times 2}$, and $R^{2} \in \mathbb{R}$ representing the covariances of the process and measurement noises. In practice, the noise statistic is correlated with the operating environment of the battery, which is generally unknown.

\subsection{Observability Analysis}

Referring to [27], the battery model (4) satisfies the observability condition, if the following observability matrix

$$
\vartheta=\left[\begin{array}{c}
C(k) \\
C(k+1) A(\theta)
\end{array}\right]
$$

with

$$
C(k)=\left[\frac{\mathrm{d} g\left(x_{1}(k)\right)}{\mathrm{d} x_{1}} \quad-1\right]
$$

is full rank for all $x$.

As $C_{b}$ is much larger than the value of $I_{B}(k)$, the SOC change is small enough in each sampling step. Hence, $\frac{\mathrm{d} g\left(x_{1}(k)\right)}{\mathrm{d} x_{1}}$ can be simplified to a constant $k_{0}>0$ [28,29]. By substituting (4) into (6), it can be derived that

$$
\vartheta=\left[\begin{array}{cc}
k_{0} & -1 \\
k_{0} & -\theta_{1}
\end{array}\right]
$$

Since $k_{0}$ is a positive constant and $\theta_{2}$ is less than 1 as $\theta_{2}$ is an exponential function, $\vartheta$ is full rank. Hence, it can be concluded that if the model parameter vector $\theta$ is known, the battery system is observable, therefore the battery's SOC can be estimated.

The model parameters are assumed to be known in numerous SOC estimation algorithms in the literature such as [9-14]. However, the RC parameters $\theta$ in the equivalent circuit model of the battery should be identified through cumbersome and inconvenient prior experiments [30]. It motivates us to propose an SOC estimation strategy by treating the parameters $\theta$ in (4) as unknown, and online estimating the model parameters and the SOC of the battery simultaneously.

\section{Battery SOC and Model Parameter Estimation}

Based on the model developed above, an RLS algorithm is utilized for the online model parameter identification, and an adaptive SRUKF algorithm is designed to estimate the SOC of the battery.

\subsection{Model Parameter Identification with Rls Algorithm}

Referring to [28,29], it can be assumed that the OCV of the battery remains constant during a short sampling period that satisfies $g\left(x_{1}(k)\right)-g\left(x_{1}(k-1)\right)=0$. With defining $y_{1}(k)=y(k)-y(k-1)$ and $u_{1}(k)=u(k)-u(k-1)$, from (4), it yields

$$
y_{1}(k)=U^{T}(k) \Phi+v_{1}(k),
$$


with

$$
\begin{gathered}
\Phi \triangleq\left[\theta_{2},-\theta_{3}, \theta_{2} \theta_{3}-\left(1-\theta_{2}\right) \theta_{1}\right]^{T} \\
U(k) \triangleq\left[y_{1}(k-1), u_{1}(k), u_{1}(k-1)\right]^{T},
\end{gathered}
$$

where $v_{1}(k)$ is the noise. Then, an RLS is proposed for the battery model parameters online identification as follows:

$$
\hat{\Phi}(k)=\hat{\Phi}(k-1)+\Gamma(k)\left(y_{1}(k)-U^{T}(k) \Phi(k-1)\right)
$$

where $\hat{\Phi}(k)=\left[\hat{\phi}_{1}(k), \hat{\phi}_{2}(k), \hat{\phi}_{3}(k)\right]^{T} \in \mathbb{R}^{3}$ is the estimation of $\Phi ; \Gamma(k) \in \mathbb{R}^{3}$ is the designed gain vector that can be updated as

$$
\begin{aligned}
& \Gamma(k)=\frac{P(k-1) U(k)}{\lambda+U^{T}(k) P_{\theta}(k-1) U(k)} \\
& P_{\theta}(k)=\frac{P_{\theta}(k-1)-\Gamma(k) U^{T}(k) P_{\theta}(k-1)}{\lambda},
\end{aligned}
$$

with $\lambda$ denoting the forgetting factor that is normally chosen as a value between 0.98 and 0.995 . Based on (9) and (10), the estimated battery model parameter vector $\hat{\theta}(k)$ is calculated that

$$
\hat{\theta}(k)=\left[\frac{-\hat{\phi}_{1}(k) \hat{\phi}_{2}(k)-\hat{\phi}_{3}(k)}{1-\hat{\phi}_{1}(k)}, \hat{\phi}_{1}(k),-\hat{\phi}_{2}(k)\right]^{T} .
$$

\subsection{Adaptive Square-Root Ukf Based Soc Estimation}

Different from the traditional UKF, the square-root of the state covariance matrix is directly propagated in the SRUKF method, avoiding the need to calculate the square-root of the state covariance at each iteration step. The SRUKF is more advantageous with better numerical properties and guaranteed positive semi-definiteness of the state covariance matrix [31]. Due to these advantages, based on the battery model with the estimated model parameters, an adaptive SRUKF algorithm is adopted here to estimate the battery's SOC, where an adaptive noise statistic estimator is utilized to update the noise statistic information at each sampling instant to get rid of its dependence on priori noise information. The algorithmic process of the adaptive SRUKF algorithm can be divided into four steps: initialization, prediction, measurement update, and noise statistic estimation, which are provided as follows:

Step 1: Initialization. The initial estimated state vector is set as $\hat{x}(0)=E[x(0)]$ and the initial square-root of the state covariance matrix is $S(0)=\operatorname{cholupdate}\left\{E\left[(x(0)-\hat{x}(0))(x(0)-\hat{x}(0))^{T}\right]\right\}$, where $E[\cdot]$ denotes the mean and cholupdate $\{\cdot\}$ represents the Cholesky factorization [32]. The initial estimated covariances of the process and measurement noises are set as $\hat{Q}^{2}(0)=Q_{0}^{2}$ and $\hat{R}^{2}(k)=$ $R_{0}^{2}$, respectively.

Step 2: Prediction. The UT technique is utilized for the propagation of the states' mean and covariance, where sigma points are chosen to approximate the mean and the covariance of the actual distribution. A set of $2 n+1$ sigma points are selected in the following way

$$
\begin{cases}\chi_{0}(k-1)=\hat{x}(k-1), & \\ \chi_{i}(k-1)=\hat{x}(k-1)+\left(\sqrt{n+\lambda} S_{i}(k-1)\right), & i=1, \cdots, n, \\ \chi_{i}(k-1)=\hat{x}(k-1)-\left(\sqrt{n+\lambda} S_{i-n}(k-1)\right), & i=n+1, \cdots, 2 n\end{cases}
$$

where $\hat{x}(k-1) \in \mathbb{R}^{n}$ denotes the estimated state vector at time step $k-1, n$ represents the state vector's dimension, $S_{i}(k-1)(1 \leq i \leq n)$ is the $i$-th column of the matrix $S(k-1) \in \mathbb{R}^{n \times n}$ with $S(k-1)$ the square-root matrix of the state covariance, $\lambda$ is a scalar that can be calculated as

$$
\lambda=\alpha^{2}(n+\sigma)-n
$$


where $\alpha$ and $\sigma$ are the tuning parameters determining the spread of the sigma points. With utilizing $\hat{\theta}(k-1)$ to replace $\theta$ in (4), each point in (12) is updated through the battery model as

$$
\chi_{i}(k \mid k-1)=A(\hat{\theta}(k-1)) \chi_{i}(k-1)+B(\hat{\theta}(k-1)) u(k-1),
$$

for $0 \leq i \leq 2 n$. Then, the the a priori estimation of the state vector $\hat{x}(k \mid k-1)$ is computed as follows:

$$
\hat{x}(k \mid k-1)=\sum_{i=0}^{2 n} W_{i}^{(m)} \chi_{i}(k \mid k-1)
$$

with the weights

$$
\left\{\begin{array}{l}
W_{0}^{(m)}=\frac{\lambda}{\lambda+n}, \\
W_{i}^{(m)}=\frac{1}{2(\lambda+n)} \quad i=1, \cdots, 2 n .
\end{array}\right.
$$

The a priori square-root of the state covariance $S(k \mid k-1)$ is updated as:

$$
\begin{gathered}
S_{1}(k \mid k-1)=\operatorname{qr}\left\{\left[\sqrt{W_{1}^{(c)}}\left(\chi_{1}(k \mid k-1)-\hat{x}(k \mid k-1)\right) \cdots\right.\right. \\
\left.\left.\sqrt{W_{2 n}^{(c)}}\left(\chi_{2 n}(k \mid k-1)-\hat{x}(k \mid k-1)\right) \hat{Q}(k)\right]^{T}\right\} \\
S(k \mid k-1)=\text { cholupdate }\left\{S_{1}^{T}(k \mid k-1), \chi_{0}(k \mid k-1)-\hat{x}(k \mid k-1), W_{0}^{(c)}\right\}
\end{gathered}
$$

with the weights

$$
\left\{\begin{array}{l}
W_{0}^{(c)}=\frac{\lambda}{\lambda+n}+\left(1-\alpha^{2}+\beta\right), \\
W_{i}^{(c)}=\frac{1}{2(\lambda+n)} \quad i=1, \cdots, 2 n
\end{array},\right.
$$

where $q r\{\cdot\}$ denotes the $\mathrm{QR}$ decomposition [32]; $\hat{Q}(k)$ denotes the estimation of the square-root of the process noise covariance matrix; $\beta$ is a constant, which is usually chosen as 2 for the Gaussian distribution of the states [33]. Note that the QR decomposition of the matrix $D=\left[\sqrt{W_{1}^{(c)}}\left(\chi_{1}(k \mid k-1)-\right.\right.$ $\hat{x}(k \mid k-1)) \cdots \hat{Q}(k-1)]^{T} \in \mathbb{R}^{3 n \times n}$ is given by $D=Q_{1} R_{1}$, where $Q_{1} \in \mathbb{R}^{3 n \times 3 n}$ is an orthogonal matrix and $R_{1} \in \mathbb{R}^{3 n \times n}$ is an upper triangular matrix [32]. Here, $\operatorname{qr}\{D\}$ denotes the QR decomposition of the matrix $D$ with $\bar{R}_{1} \in \mathbb{R}^{n \times n}$ returned, where $\bar{R}_{1}$ is the upper triangular part of $R_{1}$, i.e., $S_{1}(k \mid k-1)=\bar{R}_{1}$ in (16). The Cholesky factor of the rank 1 update $P \pm v \xi \xi^{T}$ is represented as $S_{2}=$ cholupdate $\left\{S_{1}, \xi, \pm v\right\}$, where $S_{1}$ is the original Cholesky factor of $P$ with $P=S_{1} S_{1}^{T}$. In (16), $S(k \mid k-1)=$ cholupdate $\{\cdot\}$ denotes the Cholesky factor of $S_{1}^{T}(k \mid k-1) S_{1}(k \mid k-1)+W_{0}^{(c)} \xi \xi^{T}$ with $\xi=\chi_{0}(k \mid k-1)-\hat{x}(k \mid k-1)$. Note that $S_{1}^{T}(k \mid k-1)$ is used in (16) since $\bar{R}_{1}$ denotes the transpose of the Cholesky factor of $D D^{T}$ with $\bar{R}_{1}^{T} \bar{R}_{1}=\bar{R}_{1}^{T} Q^{T} Q \bar{R}_{1}=D D^{T}$. From (16), it yields that the corresponding priori sate covariance matrix $P(k \mid k-1)$ is

$$
\begin{aligned}
P(k \mid k-1) & =S(k \mid k-1) S^{T}(k \mid k-1)=D^{T} D+W_{0}^{(c)} \xi \xi^{T} \\
& =\sum_{i=0}^{2 n} W_{i}^{(c)}\left(\chi_{i}(k \mid k-1)-\hat{x}(k \mid k-1)\right)\left(\chi_{i}(k \mid k-1)-\hat{x}(k \mid k-1)\right)^{T}+\hat{Q}^{2}(k),
\end{aligned}
$$

which is consistent with that of the UKF [24]. Note that $S(k \mid k-1)$ is utilized rather than the square-root of $P(k \mid k-1)$ in the designed SRUKF since it is more efficient to calculate the QR decomposition and Cholesky factorization in (16) than performing the square-root of $P(k \mid k-1)$ directly in the UKF [15]. The estimated output is computed by propagating the sigma points based on the model (4) as follows:

$$
\hat{y}(k \mid k-1)=\sum_{i=0}^{2 n} W_{i}^{(m)} Y_{i}(k \mid k-1),
$$


with

$$
\mathrm{Y}_{i}(k \mid k-1)=h\left(\chi_{i}(k \mid k-1), \hat{\theta}(k), u(k)\right),
$$

where $\hat{y}(k \mid k-1)$ is the estimated output through the SRUKF, and $\hat{\theta}(k)$ are updated based on (9)-(11).

Step 3: Measurement update. Similarly, the square-root of the measurement covariance $S_{y}(k)$ can be calculated by

$$
S_{y}(k)=\text { cholupdate }\left\{\left[S_{y 1}^{T}(k), \mathrm{Y}_{0}(k \mid k-1)-\hat{y}(k \mid k-1), W_{0}^{(c)}\right]\right\},
$$

with

$$
S_{y 1}(k)=\operatorname{qr}\left\{\left[\sqrt{W_{1}^{(c)}}\left(\mathrm{Y}_{1}(k \mid k-1)-\hat{y}(k \mid k-1)\right) \cdots \sqrt{W_{2 n}^{(c)}}\left(\mathrm{Y}_{2 n}(k \mid k-1)-\hat{y}(k \mid k-1)\right) \hat{R}(k)\right]^{T}\right\},
$$

with $\hat{R}(k)$ denoting the estimated square-root of the measurement noise covariance. The cross-correlation covariance $P_{x y}(k)$ are obtained as

$$
P_{x y}(k)=\sum_{i=0}^{2 n} W_{i}^{(c)}\left[\chi_{i}(k \mid k-1)-\hat{x}(k \mid k-1)\right]\left[Y_{i}(k \mid k-1)-\hat{y}(k \mid k-1)\right] .
$$

Then, the estimated state vector $\hat{x}(k)$ is updated as follows:

$$
\hat{x}(k)=\hat{x}(k \mid k-1)+L(k)(y(k)-\hat{y}(k \mid k-1)),
$$

with the filter gain vector $L(k)$ updated as

$$
L(k)=P_{x y}(k)\left(S_{y}^{-1}(k)\right)^{T} S_{y}^{-1}(k),
$$

where $S_{y}^{-1}(k)$ denotes the inverse of $S_{y}(k)$. Based on (20) and (24), the square-root matrix of the state covariance is updated that

$$
S(k)=\text { cholupdate }\left\{S(k \mid k-1), L(k) S_{y}(k),-1\right\} .
$$

Step 4: Noise statistic estimation. A moving window of the output estimation error sequence is utilized for the noise statistic estimation. Referring to [14,24], the covariances of the process and measurement noises are updated as

$$
\begin{aligned}
\hat{Q}^{2}(k)=L(k)\left(\sum_{j=k-L+1}^{k}(y(j)-\hat{y}(j \mid j-1))(y(j)-\hat{y}(j \mid j-1))^{T}\right) L^{T}(k) \\
\hat{R}^{2}(k)=\sum_{j=k-L+1}^{k}(y(j)-\hat{y}(j \mid j-1))(y(j)-\hat{y}(j \mid j-1))^{T} \\
\quad+\sum_{i=1}^{2 n} W_{i}^{(c)}\left(\mathrm{Y}_{i}(k \mid k-1)-y(k)\right)\left(\mathrm{Y}_{i}(k \mid k-1)-y(k)\right)^{T},
\end{aligned}
$$

where $L$ is the size of the selected moving window used for covariance matching.

\section{Experimental Results}

To validate the performance of the designed adaptive SRUKF with RLS-based model parameters online identification on battery SOC estimation, an IFP36130155-36Ah lithium-ion battery with a nominal capacity of $36 \mathrm{Ah} \mathrm{[26]} \mathrm{was} \mathrm{chosen} \mathrm{for} \mathrm{the} \mathrm{experiment.} \mathrm{The} \mathrm{experiment} \mathrm{was} \mathrm{conducted} \mathrm{at} \mathrm{room}$ temperature. The mapping from the battery's SOC to its OCV was as shown in Figure 2. The ITECH dc electronics Load IT8512 was programmed to simulate the actual working condition of the battery with the recorded signals of the current and the corresponding terminal voltage shown in Figures 3 and 4, where the sampling rate is $1 \mathrm{~Hz}$. In the RLS algorithm, the initial estimated model parameter vector was randomly selected as $\hat{\theta}_{0}=[0.001,0.95,0.02]^{T}, \lambda$ and $P_{\theta}(0)$ are set as 0.99 and diag $\{0.1,0.1,0.1\}$, 
respectively. For the proposed adaptive SRUKF algorithm, the initial estimated state vector was randomly selected as $\hat{x}(0)=[70 \%, 0]^{T}$. The initial square-root state estimation covariance matrix and the initial estimated square-root covariances of the process and measurement noises were randomly set as $S_{0}=\operatorname{diag}\{0.01,0.01\}, Q_{0}=\operatorname{diag}\{0.01,0.01\}$, and $R_{0}=0.05$, respectively. The parameters $L, \alpha$, and $\beta$ were selected as $100,0.85$, and 2 , respectively.

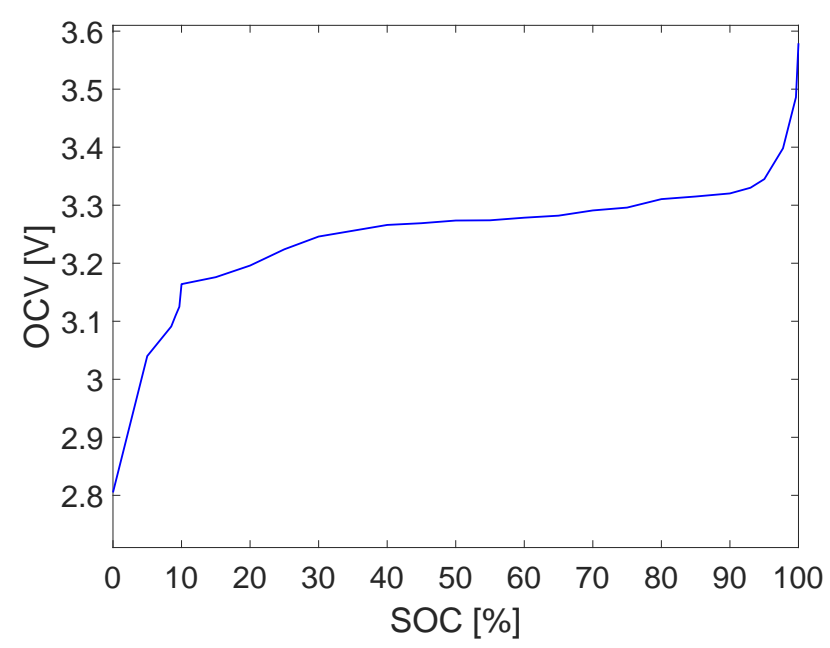

Figure 2. Open circuit voltage (OCV) versus state-of-charge (SOC) of the battery [26].

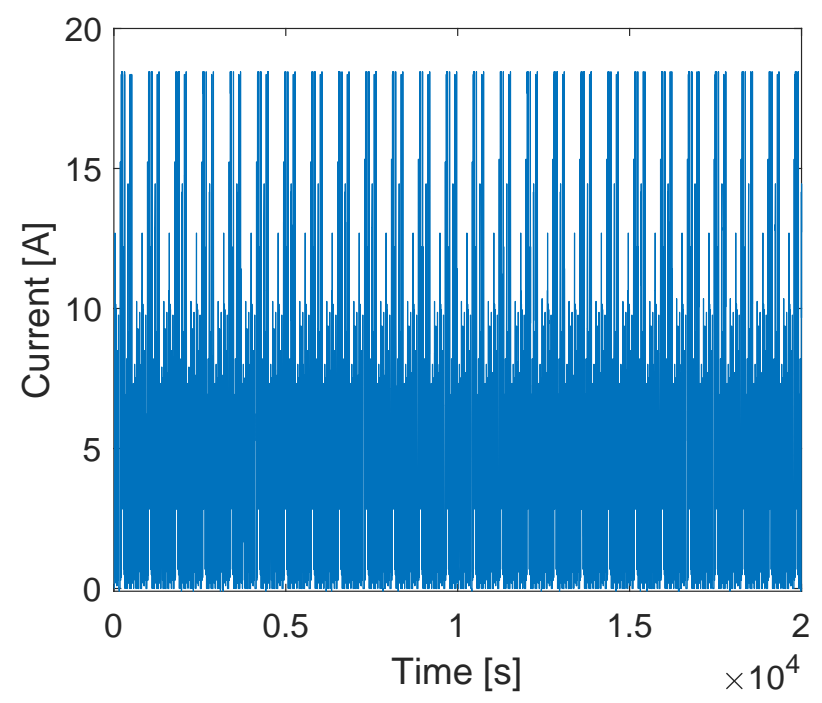

Figure 3. Applied current signal.

The experimental results in terms of the estimated model parameters $\theta_{i}(1 \leq i \leq 3)$ are demonstrated in Figures 5-7. The corresponding estimated $C_{t}$ is shown in Figure 8. As a comparison, referring to [26], the parameter vector $\theta$ in the battery equivalent circuit model is calculated offline through utilizing the least-squares method with the results of $\hat{\theta}=[0.00631,0.9838,0.0224]^{T}$. 


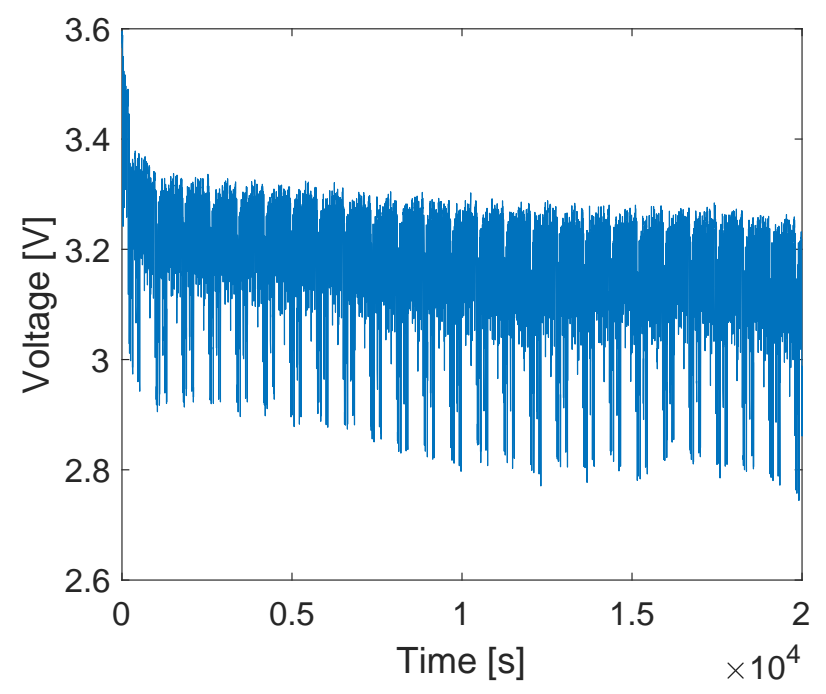

Figure 4. Terminal voltage of the battery.

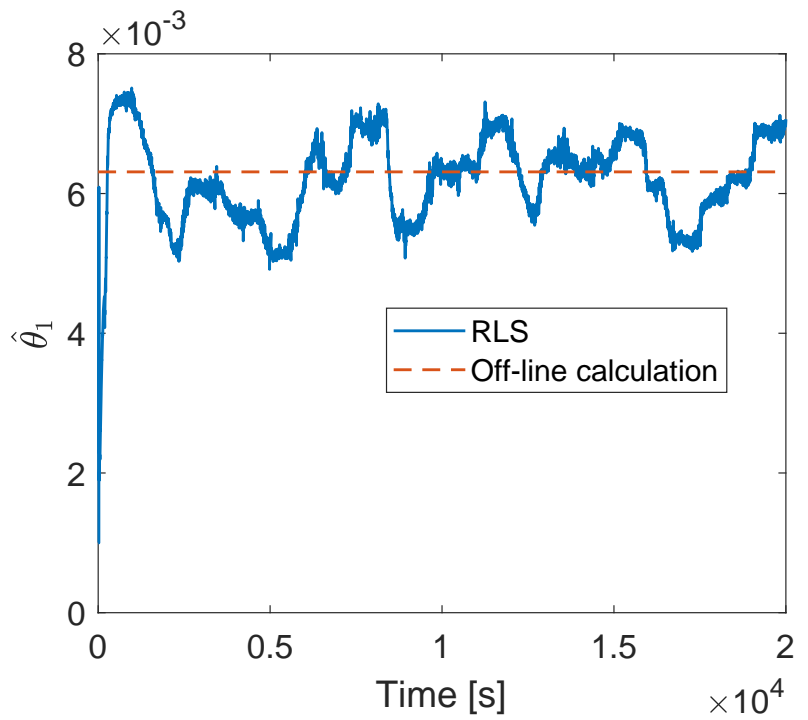

Figure 5. Comparison of the estimated results of parameter $\theta_{1}$.

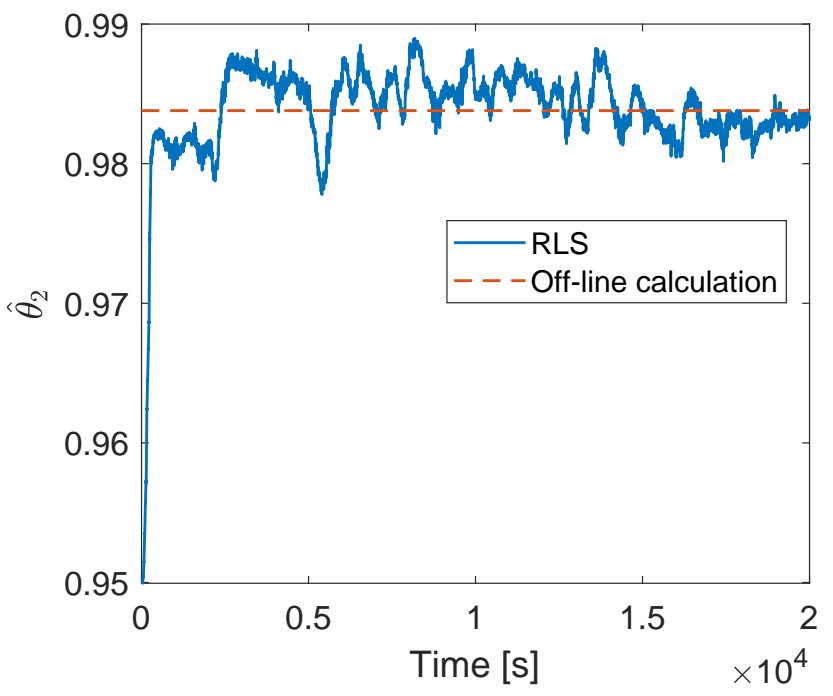

Figure 6. Comparison of the estimated results of parameter $\theta_{2}$. 


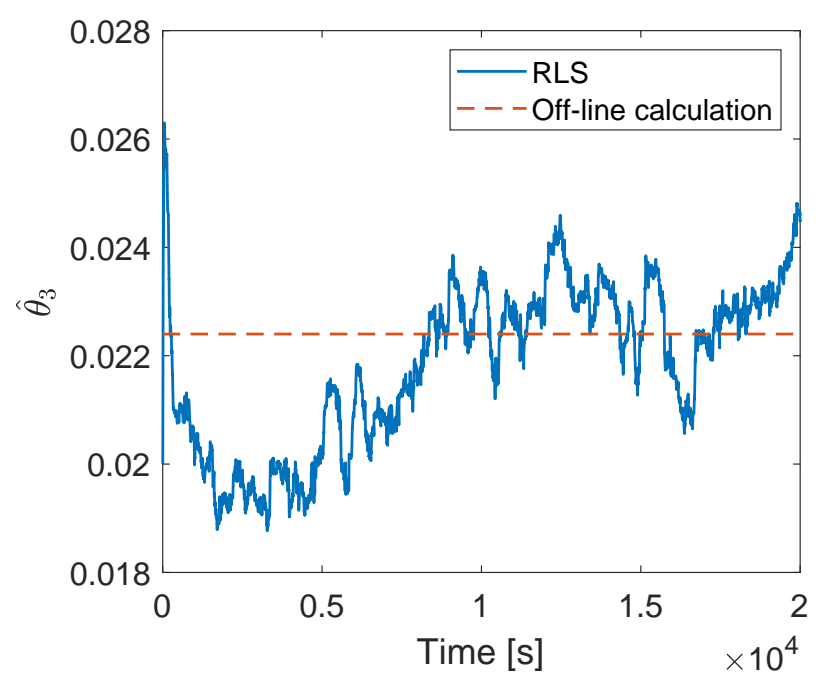

Figure 7. Comparison of the estimated results of parameter $\theta_{3}$.

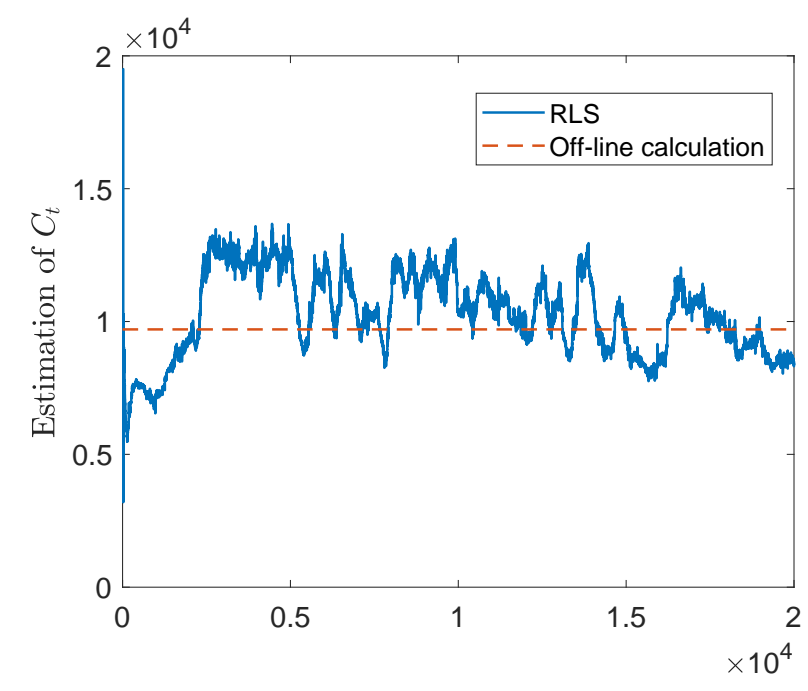

Figure 8. Comparison of the estimated results of $C_{t}$.

The actual and estimated SOCs by the adaptive SRUKF with RLS-based model parameters online identification are illustrated in Figure 9. The battery's actual SOC is gotten with using the ampere-hour counting method, where its initial SOC is obtained through measuring the initial OCV of the battery [20]. The SOC estimation error of the designed approach, calculated by subtracting the actual SOC from the estimated one, is illustrated in Figure 10. The statistic results in terms of the mean and root mean square (RMS) of the SOC estimation error are $0.13 \%$ and $0.98 \%$, respectively, in which the mean and RMS errors are calculated as follows:

$$
\begin{aligned}
& \text { mean }=\frac{1}{N} \sum_{k=1}^{N}\left|x_{1}(k)-\hat{x}_{1}(k)\right| \\
& \text { RMS }=\sqrt{\frac{1}{N} \sum_{k=1}^{N}\left(x_{1}(k)-\hat{x}_{1}(k)\right)^{2}}
\end{aligned}
$$

with $N$ denoting the data number. To demonstrate the superior performance of the designed adaptive SRUKF with RLS-based model parameters online identification method, the estimation results of the SRUKF and the adaptive EKF with model parameters identified by RLS, and the adaptive SRUKF with off-line calculated model parameters are given as comparisons. The comparison of 
the measured and estimated terminal voltages is shown in Figure 11 and Table 1. It demonstrates that the methods with model parameters online identification can provide a smaller voltage estimation error compared with those with model parameter offline calculation. Their comparison results of the SOC estimation error are listed in Figure 10 and Table 2. It shows that higher estimation accuracy can be achieved by the proposed estimation method than other commonly used Kalman filter-based methods, which demonstrates its promising performance in terms of the SOC estimation.

Table 1. Statistics of the terminal voltage simulation errors.

\begin{tabular}{ccc}
\hline & Mean & RMS \\
\hline Adaptive EKF + RLS & $0.0108 \mathrm{~V}$ & $0.0194 \mathrm{~V}$ \\
SRUKF + RLS & $0.0082 \mathrm{~V}$ & $0.0111 \mathrm{~V}$ \\
Adaptive SRUKF + offline calculation & $0.0117 \mathrm{~V}$ & $0.0635 \mathrm{~V}$ \\
Adaptive SRUKF + RLS & $0.0081 \mathrm{~V}$ & $0.0115 \mathrm{~V}$ \\
\hline
\end{tabular}

Table 2. Statistical results of the SOC estimation errors.

\begin{tabular}{ccc}
\hline & Mean & RMS \\
\hline Adaptive EKF + RLS & $1.14 \%$ & $2.22 \%$ \\
SRUKF + RLS & $1.91 \%$ & $2.38 \%$ \\
Adaptive SRUKF + offline calculation & $1.08 \%$ & $1.99 \%$ \\
Adaptive SRUKF + RLS & $0.13 \%$ & $0.98 \%$ \\
\hline
\end{tabular}

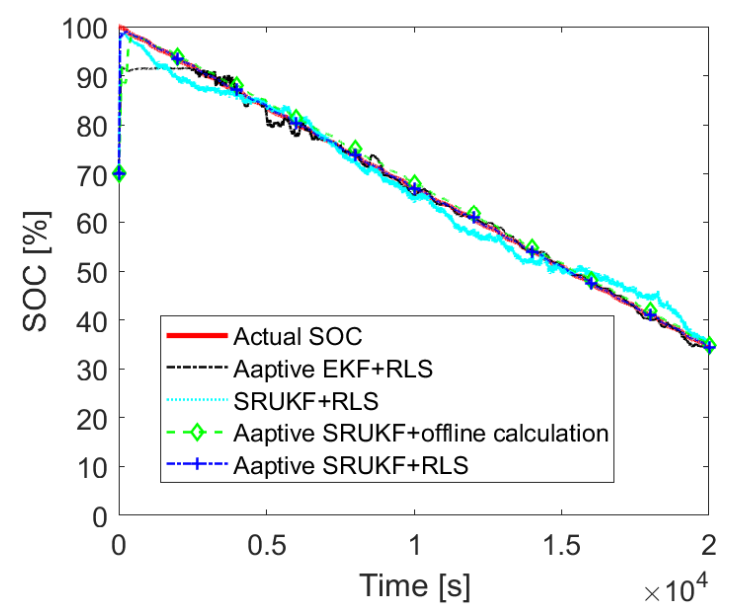

Figure 9. Actual and estimated SOC curves.

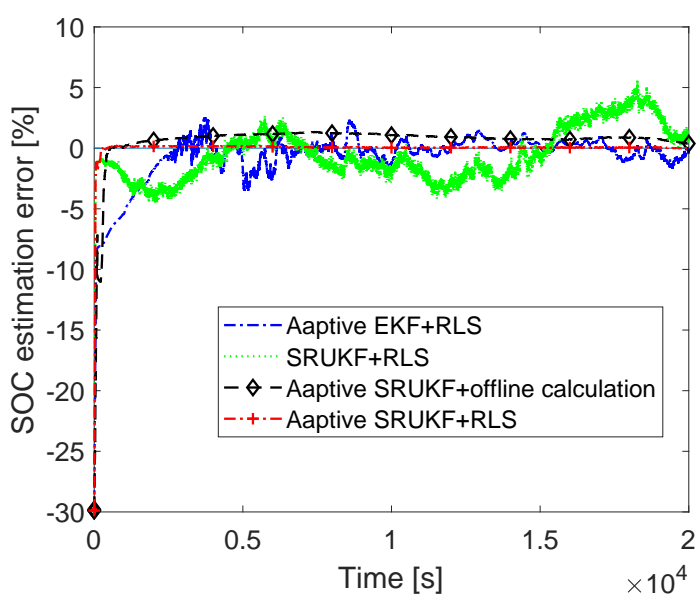

Figure 10. SOC estimation error of the battery. 


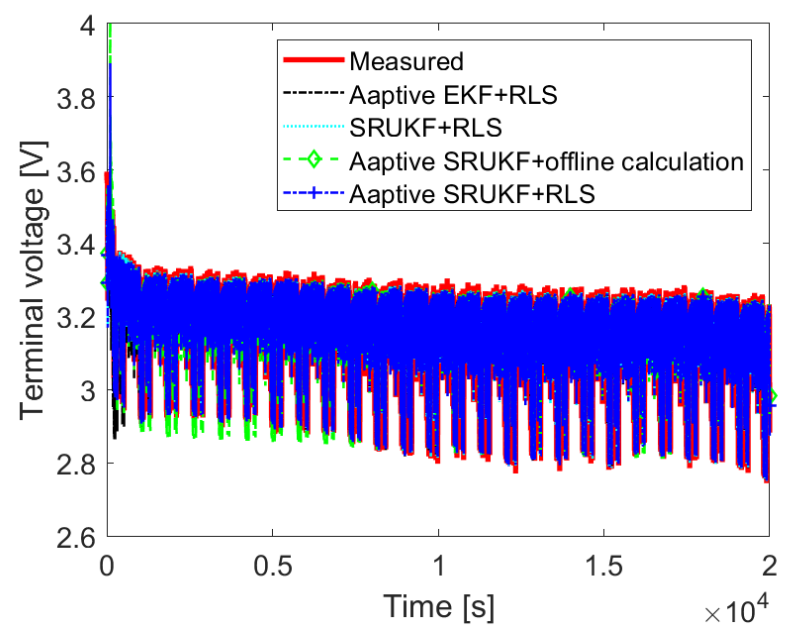

Figure 11. Comparison of the measured and simulated terminal voltages.

\section{Conclusions}

Accurate SOC estimation is important for the operation optimization of the battery. Based on a battery equivalent circuit model with unknown parameters, an RLS algorithm is utilized for model parameters online identification, and an adaptive SRUKF is proposed to estimate the battery's SOC, where a noise statistic estimator is utilized for the noise information online update. Extensive experimental results are provided to validate the performance of the proposed method showing that the mean and RMS of the SOC estimation error can be $0.13 \%$ and $0.98 \%$, respectively. Higher accuracy in the SOC estimation is demonstrated compared with other commonly-used Kalman filter-based methods.

Author Contributions: Conceptualization, Q.O. and Z.W. (Zhisheng Wang); Methodology, Q.O.; Software, R.M.; Validation, Q.O., G.X. and R.M.; Formal Analysis, Z.W. (Zhaoxiang Wu); Investigation, R.M.; Resources, Z.W. (Zhaoxiang Wu); Data Curation, G.X.; Writing-Original Draft Preparation, Q.O.; Writing-Review \& Editing, Q.O.; Visualization, R.M.; Supervision, Z.W. (Zhisheng Wang); Project Administration, Z.W. (Zhisheng Wang); Funding Acquisition, Q.O. All authors have read and agreed to the published version of the manuscript.

Funding: This work was supported in part by the National Natural Science Foundation of China (Grant No. 61903189), and in part by the Open Research Project of the State Key Laboratory of Industrial Control Technology, Zhejiang University, China (No. ICT20053).

Conflicts of Interest: The authors declare no conflict of interest.

\section{References}

1. Tsujikawa, T.; Yabuta, K.; Arakawa, M.; Hayashi, K. Safety of Large-Capacity Lithium-Ion Battery and Evaluation of Battery System for Telecommunications. J. Power Sources 2013, 244, 11-16. [CrossRef]

2. Ouyang, Q.; Han, W.; Zou, C.; Xu, G.; Wang, Z. Cell Balancing Control For Lithium-Ion Battery Packs: A Hierarchical Optimal Approach. IEEE Trans. Ind. Informatics 2020, 16, 5065-5075. [CrossRef]

3. Cheng, K.W.E.; Divakar, B.P.; Wu, H.; Ding, K. Battery-Management System(BMS) and SOC Development for Electrical Vehicles. IEEE Trans. Veh. Technol. 2011, 60, 76-88. [CrossRef]

4. Lee, S.; Kim, J.; Lee, J.; Cho, B.H. State-of-Charge and Capacity Estimation of Lithium-Ion Battery Using A New Open-Circuit Voltage versus State-of-Charge. J. Power Sources 2008, 185, 1367-1373. [CrossRef]

5. Moura, S.J.; Argomedo, F.B.; Klein, R.A. Mirtabatabaei and M. Krstic, Battery State Estimation for a Single Particle Model With Electrolyte Dynamics. IEEE Trans. Control. Syst. Technol. 2017, 25, 453-468. [CrossRef]

6. Corno, M.; Bhatt, N.; Savaresi, S.M.; Verhaegen, M. Electrochemical Model-Based State of Charge Estimation for Li-Ion Cells. IEEE Trans. Control. Syst. Technol. 2015, 23, 117-127. [CrossRef]

7. Chen, M.; Rincon-Mora, G.A. Accurate Electrical Battery Model Capable of Predicting Runtime and I-V Performance. IEEE Trans. Energy Convers. 2006, 21, 504-511. [CrossRef] 
8. Kang, L.; Zhao X.; Ma, J. A New Neural Network Model for the State-of-Charge Estimation in the Battery Degradation Process. Appl. Energy 2014, 121, 20-27. [CrossRef]

9. Chen, Z.; Fu, Y.; Mi, C.C. State of Charge Estimation of Lithium-Ion Batteries in Electric Drive Vehicles Using Extended Kalman Filtering. IEEE Trans. Veh. Technol. 2013, 62, 1020-1030. [CrossRef]

10. Domenico, D.D.; Prada, E.; Creff, Y. An Adaptive Strategy for Li-Ion Battery Internal State Estimation. Control. Eng. Pract. 2013, 21, 1851-1859. [CrossRef]

11. He, H.; Xiong, R.; Zhang, X.; Su, F. State-of-Charge Estimation of the Lithium-Ion Battery Using An Adaptive Extended Kalman Filter based on An Improved Thevenin Model. IEEE Trans. Veh. Technol. 2011, 60, 1461-1469.

12. Xiong, R.; Gong, X.; Mi, C.C.; Sun, F. A Robust State-of-Charge Estimator for Multiple Types of Lithium-Ion Batteries Using Adaptive Extended Kalman Filter. J. Power Sources 2013, 243, 805-816. [CrossRef]

13. Tian, Y.; Xia, B.; Sun, W.; Xu, Z. A Modified Model based State of Charge Estimation of Power Lithium-Ion Batteries Using Unscented Kalman Filter. J. Power Source 2014, 270, 619-626. [CrossRef]

14. Sun, F.; Hu, X.; Zou, Y.; Li, S. Adaptive Unscented Kalman Filter for State of Charge Estimation of A Lithium-Ion Battery for Electric Vehicles. Energy 2011, 36, 3531-3540. [CrossRef]

15. Huang, M.; Li, W.; Yan, W. Estimating parameters of synchronous generators using square-root unscented Kalman filter. Electr. Power Syst. Res. 2010, 80, 1137-1144. [CrossRef]

16. Gholizade-Narm, H.; Charkhgard, M. Lithium-ion battery state of charge estimation based on square-root unscented Kalman filter. IET Power Electron. 2013, 6, 1833-1841. [CrossRef]

17. Aung, H.; Low, K.S.; Goh, S.T. State-of-Charge Estimation of Lithium-Ion Battery Using Square Root Spherical Unscented Kalman Filter (Sqrt-UKFST) in Nanosatellite. IEEE Trans. Power Electron. 2015, 30, 4774-4783. [CrossRef]

18. Liu, S.; Cui, N.; Zhang, C. An Adaptive Square Root Unscented Kalman Filter Approach for State of Charge Estimation of Lithium-Ion Batteries. Energies 2017, 10, 1345.

19. Xu, J.; Mi, C.C.; Cao, B.; Deng, J.; Chen, Z.; Li, S. The State of Charge Estimation of Lithium-Ion Batteries Based on a Proportional-Integral Observer. IEEE Trans. Veh. Technol. 2014, 63, 1614-1621.

20. Chen, J.; Ouyang, Q.; Xu, C.; Su, H. Neural Network-Based State of Charge Observer Design for Lithium-Ion Batteries. IEEE Trans. Control. Syst. Technol. 2018, 26, 313-320. [CrossRef]

21. Messier, P.; Nguyen, B.; LeBel, F.; Trovao, J.P.F. Disturbance observer-based state-of-charge estimation for Li-ion battery used in light electric vehicles. J. Energy Storage 2020, 27, 101144. [CrossRef]

22. Roscher, M.A.; Bohlen, O.S.; Sauer, D.U. Reliable State Estimation of Multicell Lithium-ion Battery Systems. IEEE Trans. Energy Convers. 2011, 26, 737-743. [CrossRef]

23. Fang, H.; Wang, Y.; Sahinoglu, Z.; Wada, T.; Hara, S. State of Charge Estimation for Lithium-Ion Batteries: An Adaptive Approach. Control. Eng. Pract. 2014, 25, 45-54. [CrossRef]

24. Partovibakhsh, M.; Liu, G. An Adaptive Unscented Kalman Filtering Approach for Online Estimation of Model Parameters and State-of-Charge of Lithium-Ion Batteries for Autonomous Mobile Robots. IEEE Trans. Control. Syst. Technol. 2015, 23, 357-363. [CrossRef]

25. Chen, C.; Xiong, R.; Shen, W. A Lithium-ion Battery-in-the Loop Approach to Test and Validate Multiscale Dual H Infinity Filters for State-of-Charge and Capacity Estimation. IEEE Trans. Power Electron. 2018, 33, 332-342. [CrossRef]

26. Ouyang, Q.; Chen, J.; Zheng, J. State-of-Charging Observer Design for Batteries With On-line Model Parameter Identification: A Robust Approach. IEEE Trans. Power Electron. 2020, 35, 5820-5831. [CrossRef]

27. Reif, K.; Gunther, S.; Yaz, E.; Unbehauen, R. Stochastic Stability of the Discrete-Time Extended Kalman Filter. IEEE Trans. Autom. Control. 1999, 44, 714-728. [CrossRef]

28. Rahimi-Eichi, H.; Baronti, F.; Chow, M.Y. Online Adaptive Parameter Identification and State-of-Charge Coestimation for Lithium-Polymer Battery Cells. IEEE Trans. Ind. Electron. 2014, 61, 2053-2061. [CrossRef]

29. Chen, X.; Shen, W.; Cao, Z.; Kapoor, A. A novel approach for state of charge estimation based on adaptive switching gain sliding mode observer in electric vehicles. J. Power Sources 2014, 246, 667-678. [CrossRef]

30. Han, J.; Kim, D.; Sunwoo, M. State-of-Charge Estimation of Lead-Acid Batteries Using An Adaptive Extended Kalman Filter. J. Power Sources 2009, 188, 606-612. [CrossRef]

31. Haykin, S.S. Kalman Filtering and Neural Networks; Wiley: New York, NY, USA, 2001. 
32. Van der Merwe, R.; Wan, E.A. The square-root unscented Kalman filter for state and parameter-estimation. In Proceedings of the IEEE International Conference on Acoustics, Speech, and Signal Processing, Salt Lake City, UT, USA, 7-11 May 2001.

33. Julier, S.; Uhlmann, J.; Durrant-Whyte, H.F. A new method for the nonlinear transformation of means and covariances in filters and estimators. IEEE Trans. Autom. Control. 2000, 45, 477-482. [CrossRef]

(C) 2020 by the authors. Licensee MDPI, Basel, Switzerland. This article is an open access article distributed under the terms and conditions of the Creative Commons Attribution (CC BY) license (http:/ / creativecommons.org/licenses/by/4.0/). 